\title{
Orion Rendezvous, Proximity Operations, and Docking Design and Analysis
}

\author{
Christopher D'Souza ${ }^{1}$, F. Chad Hanak ${ }^{1}$, and Pete Spehar ${ }^{1}$. \\ NASA Johnson Space Center, Houston, TX 77058, USA \\ and \\ Fred D. Clark ${ }^{2}$ and Mark Jackson ${ }^{2}$ \\ Charles Stark Draper Laboratory, Houston, TX 77058, USA
}

\begin{abstract}
The Orion vehicle will be required to perform rendezvous, proximity operations, and docking with the International Space Station (ISS) and the Earth Departure Stage (EDS)/Lunar Landing Vehicle (LLV) stack in Low Earth Orbit (LEO) as well as with the Lunar Landing Vehicle in Low Lunar Orbit (LLO). The RPOD system, which consists of sensors, actuators, and software is being designed to be flexible and robust enough to perform RPOD with different vehicles in different environments. This paper will describe the design and the analysis which has been performed to date to allow the vehicle to perform its mission. Since the RPOD design touches on many areas such as sensors selection and placement, trajectory design, navigation performance, and effector performance, it is inherently a systems design problem. This paper will address each of these issues in order to demonstrate how the Orion RPOD has been designed to accommodate and meet all the requirements levied on the system.
\end{abstract}

\section{Introduction}

$T^{1}$

He Orion Crew Exploration Vehicle (CEV) is being designed to perform rendezvous, proximity operations and docking (RPOD) with the International Space Station (ISS), and the Lunar Landing Vehicle (LLV) / Earth Departure Stage (EDS) in Low Earth Orbit (LEO) and the LLV in Low Lunar Orbit (LLO). The Orion RPOD team has performed a GNC design for the CEV that meets the requirements levied on the RPOD system. This paper will discuss the challenges as well as describe the solutions that have been found to meet the requirements.

In Section 2, the driving requirements that affect RPOD will be discussed. Section 3 contains a description of the RPOD GNC hardware used. In Section 4, the trajectories will be described. Section 5 contains the analysis that was used to validate the design. Finally, a few concluding comments are made in Section 6. This paper is specifically concerned with the RPOD GN\&C. Other areas that touch on the RPOD design, such as FDIR, automation/autonomy, power, while important in their own right, are not covered in this paper.

\section{Driving Requirements}

Any design, for RPOD or otherwise, must be designed to meet a set of requirements; these may be high level (such as 'Do rendezvous') or may contain lower level requirements. It should come as no surprise that a system as complex as Orion has a great deal of requirements that must be met. Orion is required to rendezvous and dock with both the ISS and the LLV, the latter in both LEO and LLO. References 1 and 2 detail the overall mission architecture of Orion, particularly as it fits in within the Constellation architecture. Table 1 provides a glimpse of some of the driving GN\&C requirements that the RPOD system must satisfy.

${ }^{1}$ Aerospace Engineer, EG6, Aerosciences and Flight Mechanics Division

${ }^{2}$ Principal Member of the Technical Staff, Guidance, Navigation and Control Division 


\begin{tabular}{|c|c|}
\hline Number & Requirement \\
\hline CV0112 & $\begin{array}{l}\text { The active CEV shall perform relative navigation for rendezvous, proximity operations and docking } \\
\text { with the target vehicle. }\end{array}$ \\
\hline CV0116 & $\begin{array}{l}\text { The CEV shall determine the target vehicle relative position and relative velocity for proximity and } \\
\text { docking operations. }\end{array}$ \\
\hline CV0117 & $\begin{array}{l}\text { The CEV shall determine the target vehicle relative attitude and relative attitude rate for docking } \\
\text { operations. }\end{array}$ \\
\hline CV0121a & $\begin{array}{l}\text { The CEV shall perform automatic execution of rendezvous, proximity operations, and docking under } \\
\text { nominal conditions. }\end{array}$ \\
\hline CV0121b & $\begin{array}{l}\text { The CEV shall perform automatic execution of aborts from rendezvous, proximity operations, and } \\
\text { docking. }\end{array}$ \\
\hline CV0125 & The CEV shall dock with the target vehicle when target vehicle is in a planned control attitude hold. \\
\hline CV0126 & $\begin{array}{l}\text { The CEV shall dock with the target vehicle when the target vehicle is in an unplanned attitude with } \\
\text { rate less than } 0.4 \text { (TBR-002-056) deg/sec per axis. }\end{array}$ \\
\hline CV0128b & $\begin{array}{l}\text { The CEV shall perform rendezvous, proximity operations, and docking independent of Earth ground } \\
\text { asset over-flight constraints. }\end{array}$ \\
\hline CV0129 & $\begin{array}{l}\text { The CEV shall provide emergency any-attitude undocking with a mated vehicle attitude rate of less } \\
\text { than } 2 \mathrm{deg} / \mathrm{s} \text { (TBR-002-159) as specified in the CEV-LSAM and CEV ISS IRDs. }\end{array}$ \\
\hline CV0131 & $\begin{array}{l}\text { The CEV shall perform rendezvous, approach proximity operations, and docking with the LSAM / } \\
\text { EDS stack in Low Earth Orbit with the CEV functioning as the chaser vehicle. }\end{array}$ \\
\hline CV0132 & $\begin{array}{l}\text { The CEV shall perform contingency rendezvous and approach proximity operations with the LSAM } \\
\text { in Low Lunar Orbit with the un-crewed CEV functioning as the chaser vehicle. }\end{array}$ \\
\hline CV0133 & $\begin{array}{l}\text { The CEV shall support approach proximity operations and docking with the LSAM functioning as the } \\
\text { active chaser vehicle in Low Lunar Orbit. }\end{array}$ \\
\hline CV0134 & $\begin{array}{l}\text { The CEV shall separate and perform departure maneuver(s) from the uncrewed LSAM in Low Lunar } \\
\text { Orbit. }\end{array}$ \\
\hline CV0136 & $\begin{array}{l}\text { The CEV shall perform rendezvous, approach proximity operations, and docking with the ISS in Low } \\
\text { Earth Orbit with the CEV functioning as the chaser vehicle. }\end{array}$ \\
\hline CV0137 & The CEV shall separate and perform departure maneuver(s) from the ISS in Low Earth Orbit. \\
\hline
\end{tabular}

\section{Table 1: Driving Orion RPOD GN\&C Requirements}

\section{GNC Hardware}

The RPOD system design is predicated on having sensors that provide adequate observability of the states of the two vehicles and actuators to provide the necessary thrust to effect the rendezvous. The accuracy and the types of sensors depend on the distance between the two vehicles. The sensors are grouped into two categories: inertial sensors and relative sensors. Inertial sensors provide inertial state of the CEV. Relative sensors provide measurements of the target vehicle with respect to the CEV. Some sensors, such as the star tracker can provide both functions - they provide inertial as well as relative measurements.

\section{A. Inertial Sensors}

As the name indicates, the inertial sensors provide measurements of the CEV (navigation base) with respect to an inertial reference frame. The instruments that fall in this category include gyros, accelerometers, star trackers and GPS receivers. The current baseline includes all of these instruments. In addition, for the LEO rendezvous missions, a set of ground updates of the target vehicle are assumed when the two vehicles have ranges larger than $200 \mathrm{~km}$. It is assumed that the IMU will be operating throughout the rendezvous, from insertion through docking. When the CEV gets close to the ISS (less than $1 \mathrm{~km}$ ), it is anticipated that multi-path and blockages of the ISS will severely inhibit adequate GPS navigation data; therefore, the GPS function is disabled when the two vehicles are less than 1

2

American Institute of Aeronautics and Astronautics 
$\mathrm{km}$ apart. The exact composition of the inertial sensor suite, including the numbers of sensors is not finalized; this will be delineated prior to Preliminary Design Review (PDR) (currently scheduled for May 2008).

\section{B. Relative Sensors}

The current baseline relative sensor suite includes the use of the star tracker from $200 \mathrm{~km}$ to $5 \mathrm{~km}$ (lighting permitting), communications-based RF range and range-rate (from $30 \mathrm{~km}$ to $1 \mathrm{~km}$ for LEO) and a Vision Navigation System (VNS) (from $5 \mathrm{~km}$ to dock). The VNS will have the capability to provide range and bearing to a set of docking targets. The range and bearing to these targets are the foundation for relative attitude sensing. At a range of $50 \mathrm{ft}$, the VNS is expected to provide relative attitude measurement data. As with the inertial sensors, the exact composition of the relative sensor suite will be decided prior to PDR. The concept of operations of the relative sensor suite is described in Figure 1.

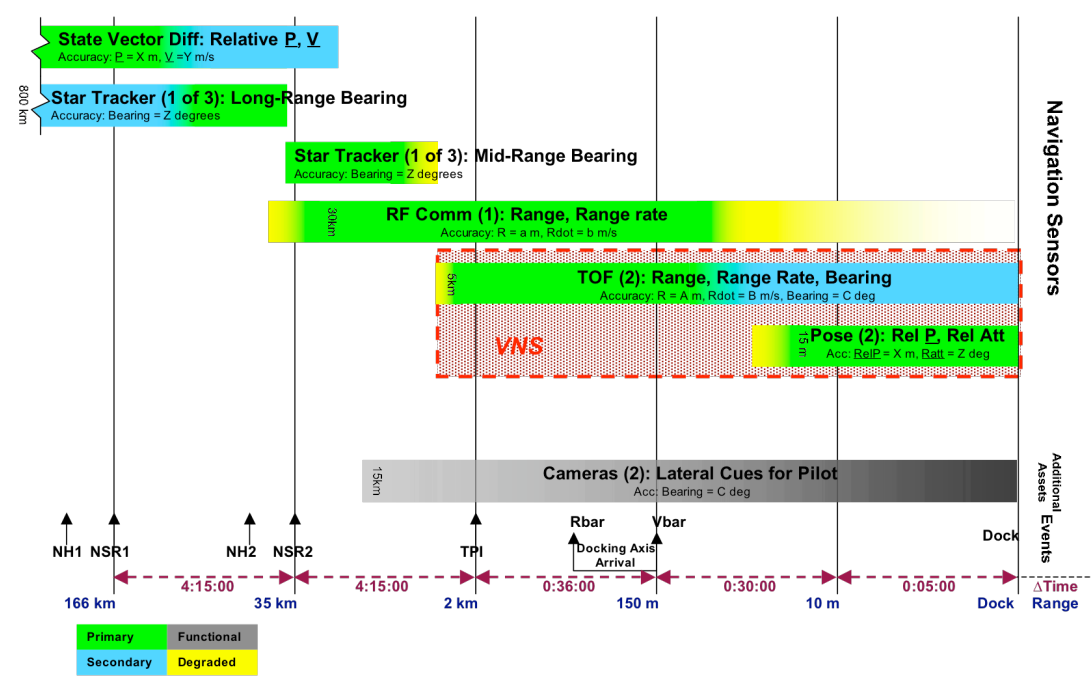

Figure 1: Orion Relative Sensor Concept of Operations

\section{Actuators}

The Orion actuators used during rendezvous and proximity operations are contained within the Service Module (SM) and commanded by the GNC software on board the CM. The actuators consist of the Orion Main Engine (OME), a reaction control system (RCS), and a set of axial reaction control thrusters (ARCS) used to back up the main engine and perform translation burns of intermediate magnitude (Figure X). Steering during OME burns is provided by thrust vector control actuators which steer the thrust vector while maintaining attitude control in the pitch and yaw axes. Roll control during OME burns is provided by the RCS. The OME is used for the insertion burn after separation from the CLV stack, as well as the trans-Earth injection maneuver (TEI) to return the CEV

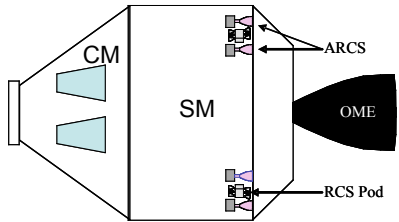

Figure 1, CEV Actuators from the Moon. The OME may also be used for larger magnitude rendezvous burns. Smaller rendezvous burns are performed by the ARCS. For these burns, the vehicle is rotated so that the longitudinal axis points in the desired burn direction, and the ARCS are fired. An off-pulsing control law is used to maintain attitude control in pitch and yaw, with RCS again providing roll control. The RCS provides attitude control during coasting operations, as well as 6 DOF control during proximity operations and docking. The RCS thrusters are arranged in four pods of six thrusters each. Thrusters are oriented according to the "Block Swap Geometry" described in Refereñe 3 . 


\section{Trajectory Design}

As stated in the introduction, the design of the trajectory cannot be divorced from the navigation system performance. After all, a trajectory design that is optimal, yet unobservable, is useless. Hence, the trajectory must be designed such that it is informed by the types of planned sensors as well as other factors. For Orion, during the LEO rendezvous, no active range or range-rate measurements are planned until the two vehicles are less than $30 \mathrm{~km}$ apart. However, bearing measurements are available as far as $200 \mathrm{~km}$. Hence, having a trajectory that does not require accurate range information would be a good system design. Additionally, having a trajectory that is passively safe in the vicinity of the target, is a highly desirable characteristic. It is precisely these factors that drove the RPOD trajectory design. The Orion RPOD design has been broken up into two major phases: the rendezvous phase and the proximity operations and docking phase. The rendezvous phase begins at insertion and ends at the TPI (Terminal Phase Initiation) maneuver that occurs at approximately $2000 \mathrm{~m}$ from the target. The proximity operations and docking phase extends from TPI to docking.

\section{A. The Rendezvous Phase}

The Orion rendezvous phase has been designed to support ISS and EDS/LLV docking on either Flight Day 1 or Flight Day 3. Trajectories have been designed to support both these options. An earlier paper (Ref 3) described the analysis that was performed to select the rendezvous trajectory. The two choices were the stable orbit rendezvous (SOR) profile flown by the Space Shuttle or a double coelliptic rendezvous profile flown on Gemini, Apollo and Skylab. A double coelliptic design was selected for the rendezvous design for several reasons. First, there is no long-range sensor that provides range and bearing (unlike the Space Shuttle that had a long-range rendezvous radar). The double coelliptic profile does not require knowledge of range to accomplish the rendezvous; rather it needs only altitude and cross-range information, both of which are provided by bearing measurements. Additionally, it provides an implicit passive safety; if the final rendezvous maneuver, the TPI maneuver, is not performed, there is no danger of collision with the target. The TPI maneuver is performed based on intersecting with a reference profile. One reported weakness of a coelliptic profile is its inability to precisely control the time of arrival (which has implications with regard to lighting considerations during proximity operations); this weakness has been mitigated by designing a double coelliptic. The altitude and the times of the coelliptic maneuvers can be adjusted to control the time of arrival. The double coelliptic trajectory for the FD3 rendezvous is depicted in Figure 2. The maneuver names, which may be somewhat strange, have been in effect at the Johnson Space Center since the first Gemini missions. The $N C$ maneuver is a phasing (catch-up) maneuver, used to adjust the downrange (or relative phase of the CEV with respect to the target) at a specific time in the future (usually one revolution later). The $N H$ is an altitude adjustment maneuver, designed to raise (or lower) the orbit of the CEV with respect to the target. The NSR is a coellipticization maneuver, designed to ensure that there is a constant differential height between the CEV and the target. The design was constrained so that the TPI maneuver occurs within 6 hours after launch. The crew workday from launch to docking was assumed to be 7 hours.

It was assumed that the CLV (Crew Launch Vehicle) would insert the CEV into a 100x-8 nmi orbit at an altitude of $70 \mathrm{nmi}$. The first maneuver, $N C_{l}$, would combine the insertion maneuver with a phasing maneuver. 

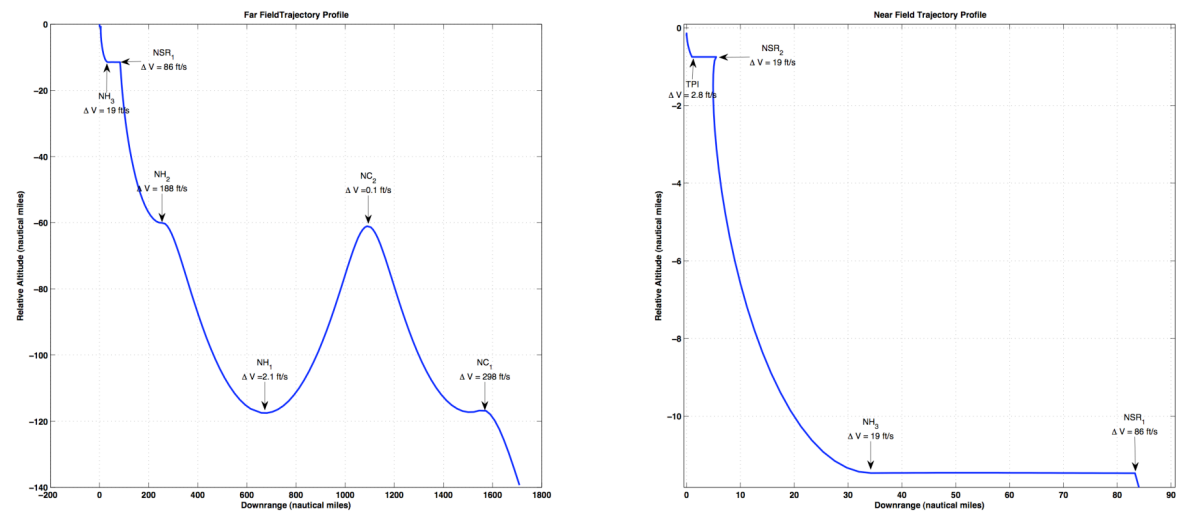

Figure 2: Flight Day 1 Rendezvous Design

A similar design has been carried out for the FD3 rendezvous design. A FD2 design was not considered due to crew considerations having to do with space adaptation.

\section{B. The Proximity Operations and Docking Phase}

Proximity operations and docking trajectories have been developed to support both CEV to ISS docking as well as docking to EDS/LLV. Since the proximity operations phase is considered to be somewhat independent of the rendezvous phase the same proximity operations profile can be used for either a FD1 or FD3 rendezvous. Because of individual vehicle structural characteristics, vehicle subsystem requirements, system limitations, operational and safety constraints, profile design considerations were made not only on relative position and velocity but also relative attitude, and attitude rates during proximity operations.

Two CEV compatible docking ports will be based on ISS, the first pointing in the direction of the ISS velocity vector (or + Vbar) mounted to Pressurized Mating Adaptor 2 (PMA-2), the second pointing towards Earth (or along the +Rbar) mounted to PMA-3. Since the ISS operational docking attitudes are limited, two separate approach trajectories were developed to accommodate docking to either PMA-2 or PMA-3 for the ISS missions. EDS/LLV will carry a single docking port that will be located on the end of the LLV and pointing along the +Rbar, similar to the PMA-3 docking port on ISS. Since the profile developed for EDS/LLV proximity operations and docking closely resembles the ISS PMA-3 docking port profile, only EDS/LLV differences from ISS profiles will be indicated in this discussion.

The proximity operations phase, as defined for the CEV, begins with the TPI maneuver and is initiated at a point on the final coelliptic phase of the rendezvous. During this period just prior to TPI through docking the CEV attitude will be such that the $+\mathrm{X}$ body axis will always be pointing towards the target vehicle. This enables crew viewing out of the forward CEV windows as well as relative sensor visibility of the target vehicle for sensor tracking. The TPI maneuver is targeted to transfer the CEV to an offset point $300 \mathrm{ft}$ behind and $1000 \mathrm{ft}$ below the target vehicle, arriving with zero relative translational rates. Having an offset point allows the CEV to be passively safe with respect to the target vehicle in the event that all CEV translational capability is lost after execution of the TPI maneuver. In this event, even with trajectory dispersions, the CEV's coasting trajectory will remain below the target vehicle's altitude and eventually phase in front of the target vehicle. This design satisfies the ISS safety requirement that the CEV trajectory does not intercept an imaginary spherical boundary (200 meter radius) around the ISS in fewer than four orbits after execution of the TPI maneuver.

The maneuver that is performed upon arrival at the offset point is called the transition to docking axis (TDA) maneuver. This maneuver is designed to transfer the CEV to the axis of the target vehicle docking port. For ISS proximity operations a common TDA point provides for flexibility in transitioning to either the PMA-2 or PMA-3

5

American Institute of Aeronautics and Astronautics 
docking port axis. The TDA2 maneuver transfers to the PMA-2 docking axis whereas the TDA3 transfers CEV to the PMA-3 docking port axis. The CEV arrival location on the docking axis is called Acquisition of the Docking Axis (ADA) point and like the TDA maneuvers is numbered according to the docking port PMA that it is approaching. ADA2 and ADA3 arrival is on the PMA-2 and PMA-3 docking axis respectively. Since the TDA2 maneuver was designed to limit CEV RCS plume impingement towards the ISS the required maneuver deltavelocity vector is mostly orthogonal to the line of sight vector between the CEV and ISS. The transfer from TDA2 to ADA2 allows for adequate clearances with ISS, is completed in about one-sixth of an orbit, and arrives at approximately $600 \mathrm{ft}$ along the PMA-2 docking axis. At docking axis arrival the ADA2 maneuver nulls the ZLVLH velocity and slight increases the closure rate to begin the final approach down the docking axis. In order to minimize potential plume impingement the TDA2 maneuver constrains the a Z-LVLH position and a X-LVLH velocity at arrival on the PMA-2 docking axis leaving the X-LVLH position and Z-LVLH velocity unconstrained. The effect of this targeting ensures that the ADA2 maneuver will require thrusting mostly orthogonal to the docking axis. After acquiring the docking axis the CEV maintains a constant closing rate of $0.3 \mathrm{ft} / \mathrm{sec}$ until a range of $30 \mathrm{ft}$ where the range rate is reduced to $0.1 \mathrm{ft} / \mathrm{sec}$ and held constant until docking contact. The proximity operations design for CEV approach to the PMA-2 docking port on ISS is depicted in Figure 3.

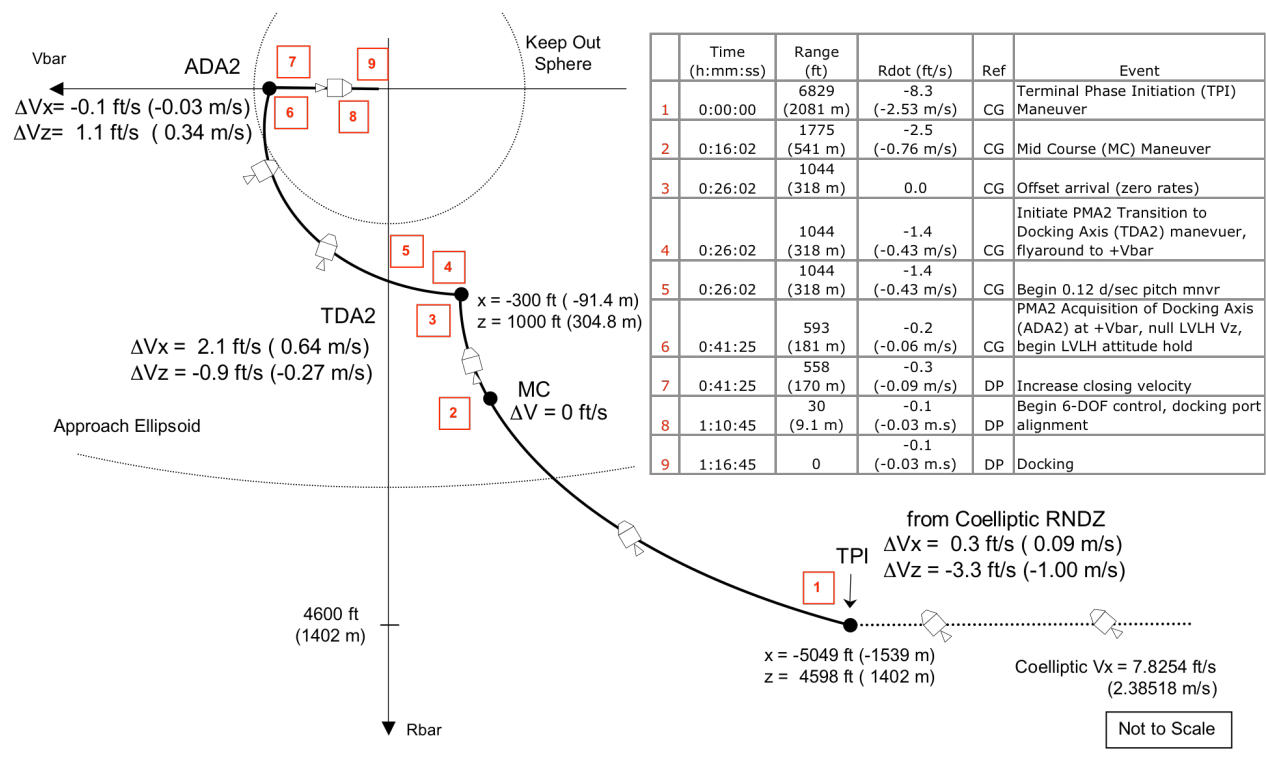

Figure 3: ISS PMA-2 Proximity Operations Design

For PMA-3 docking, the range rate profile during approach along the docking axis is designed to take advantage of the relative orbital dynamics accelerations and therefore is not constant. The nominal profile is designed so that the range rate will naturally bleed down to $0.1 \mathrm{ft} / \mathrm{sec}$ at a range of $30 \mathrm{ft}$ prior to docking to the PMA-3 docking port. This eliminates the need to fire CEV jets towards the target vehicle to slow the relative velocity, thus reducing plume impingement. To accommodate this the TDA3 maneuver was designed to deliver the CEV tangent to the docking axis with the proper closing velocity at the arrival at the ADA3 point. At ADA3 all that is required by CEV is minimal lateral translational adjustments to stay on or near the docking axis. After ADA3, the CEV will perform a $0.5 \mathrm{deg} / \mathrm{sec} 180 \mathrm{deg}$ roll maneuver to achieve proper clocking of the CEV docking port with respect to the port attached to PMA-3. The proximity operations design for CEV approach to the PMA-3 docking port on ISS is depicted in Figure 4. 


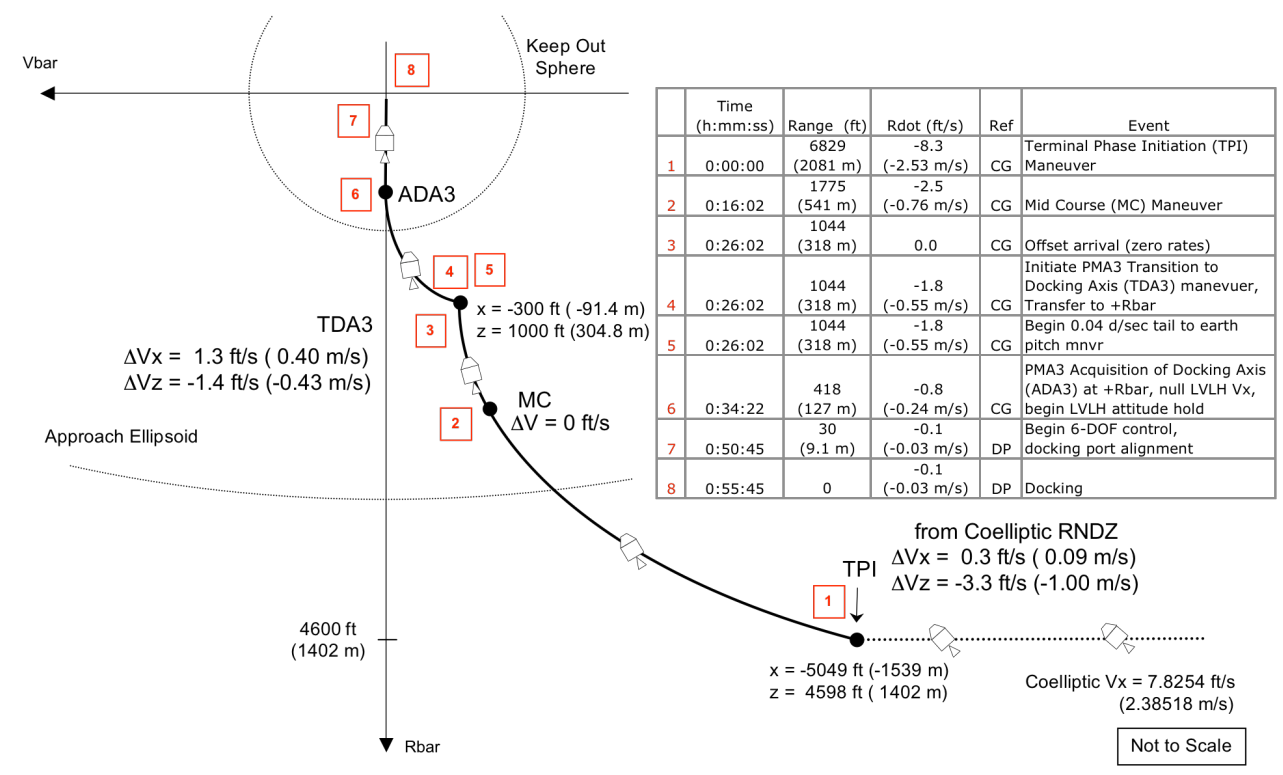

Figure 4: ISS PMA-3 Proximity Operations Design

During the final segment of the approach along the docking axis, the CEV controls its docking port position, velocity, attitude, and attitude rate relative to the target vehicle. Within a range of $50 \mathrm{ft}$ the CEV pose relative navigation sensor is used to determine lateral position and angular misalignments between the docking ports. If necessary, the CEV station-keeps at $30 \mathrm{ft}$ to perform a docking port relative angular alignment maneuver to minimize angular misalignment for docking contact. After the misalignment constraints for the docking mechanism are met the CEV continues with the final approach remaining within the docking mechanism capture envelope until docking contact.

The proximity operations design for CEV approach to EDS/LLV in LEO assumes that EDS/LLV will be holding an attitude that orients its docking port towards Earth. As a result, the CEV will perform a + Rbar approach very similar to the CEV to ISS PMA-3 approach. In this case, the main difference is that the CEV will not be required to perform the 180 deg roll maneuver after acquisition of the docking axis (ADA). The proximity operations design for CEV approach to EDS/LLV is depicted in Figure 5. 


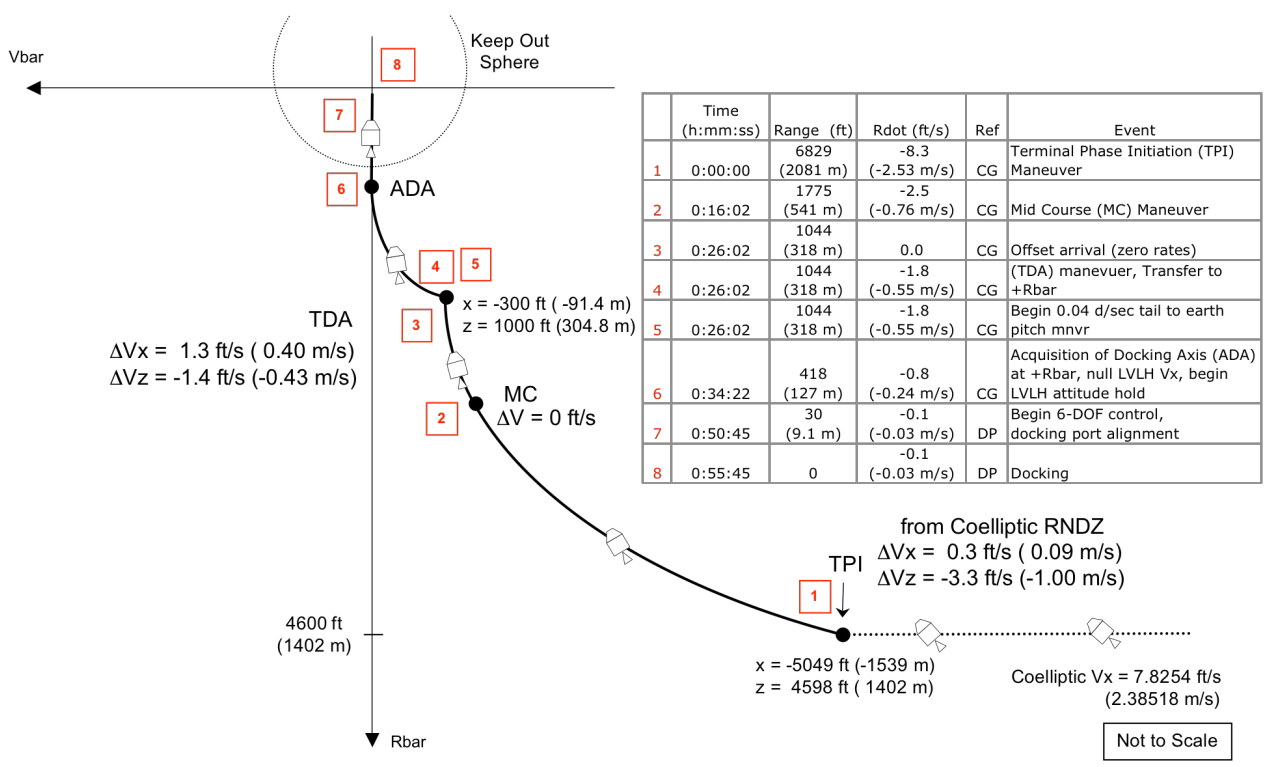

Figure 5: EDS/LLV Proximity Operations Design

\section{Analysis of Results}

An extensive set of analyses have been performed on both the rendezvous and the proximity operations and docking phases of the mission. A few of the significant results are presented below to give the reader a taste of the scope and extent of the analysis performed to date. This analysis will focus on the CEV ISS mission.

\section{A. Rendezvous Results}

In order to validate the performance of the GNC system during the rendezvous phase, a linear covariance analysis was performed. Linear covariance analysis is a methodology to obtain the navigation performance (statistics) of a system in a single run! It is a complement to Monte Carlo analysis in that it provides the statistical behavior of a GNC system without the thousands of Monte Carlo runs needed in order to truly represent the statistics of a complex dynamical system. During the early phase of a system design it is invaluable in being able to provide the engineer with the system performance, especially as it relates to navigation and guidance issues. It is used to quickly (and accurately) understand how various navigation sensors affect the navigation accuracy of a particular system design under consideration. Additionally, it also provides trajectory dispersion information (the difference between the actual trajectory and the nominal trajectory) along with variation in fuel (or $\Delta V$ ) usage. Finally, it is the perfect tool in order to trade the performance of different sensors or to determine the contribution of a particular sensor (error) parameter. For Orion, the linear covariance tool was exercised in all these many facets.

For the rendezvous analysis, the CEV was modeled with both translation and attitude states. The ISS was modeled with just the translation states. The IMU model contained 18 states, and the star tracker was modeled using three misalignment bias states. The GPS receiver measurements were modeled with six Markov states. The LIDAR sensor was modeled with three misalignments and three sensor biases states. At a range of $30 \mathrm{~km}$, the RF system acquires the ISS and provides range and range-rate measurements. These were modeled in the LINCOV tool and were modeled with two additional states, for the biases in the range and the range-rate measurements. In addition, there were 18 bias states to represent the maneuver execution error model, to account for different thrusters used for the larger and smaller maneuvers. Ground updates were modeled as discrete events prior to maneuvers when no onboard relative sensors were within range. As can be seen, the model used for this analysis was quite extensive.

8

American Institute of Aeronautics and Astronautics 
Figure 6 contains the relative navigation performance. One can see where the ground updates occur (prior to $\mathrm{NC}_{2}$ and $N H_{l}$ ) and when the star trackers and the range and range-rate and LIDAR measurements come on-line.
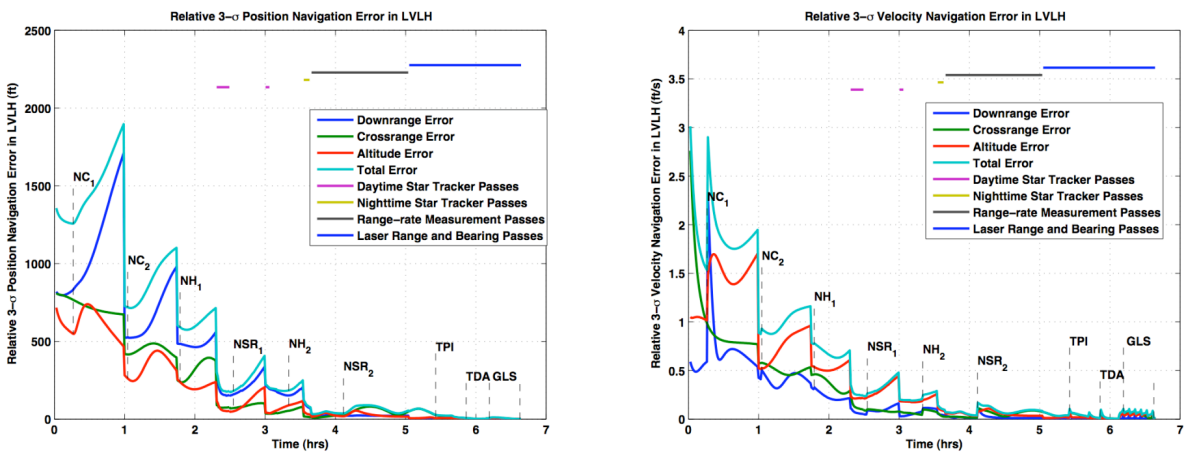

Figure 6: Flight Day 1 Relative Navigation Position and Velocity Errors

A major focus of the rendezvous analysis to date has been to analyze the performance of the LIDAR system and understand the various contributors to the total navigation error. To this end, an error breakdown has been performed to analyze the various LIDAR error contributors. Figure 7 contains the error breakdown from $5 \mathrm{~km}$ to a few meters away from ISS.
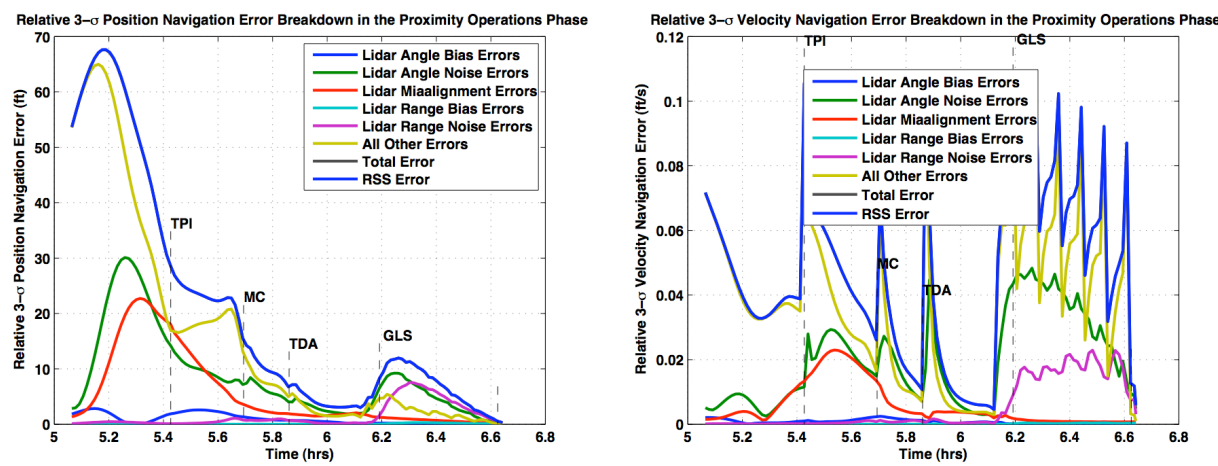

Figure 7: Flight Day 1 Relative Navigation Error Breakdown for the LIDAR

One of the major areas of concern with regard to the rendezvous performance has to do with the trajectory dispersions. Trajectory dispersions are defined as deviations of the state from the nominal trajectory. These dispersions can be expressed as position and velocity dispersions as a function of time (as in Figures 6 and 7) or they can be superimposed on the trajectory. Figure 8 contains the time history of the position and velocity dispersions, and Figure 9 contains the $3 \sigma$ position dispersions as a function of the nominal state. Figure 9 contains the in-plane trajectory dispersions as a function of the three phases of rendezvous - farfield, midfield and nearfield. 

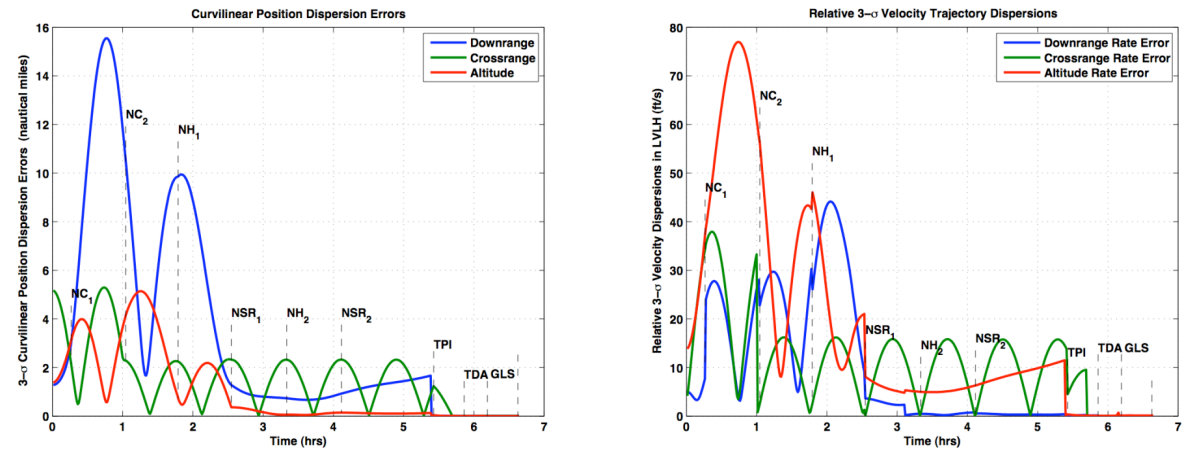

Figure 8: Flight Day 1 Relative Dispersion Errors
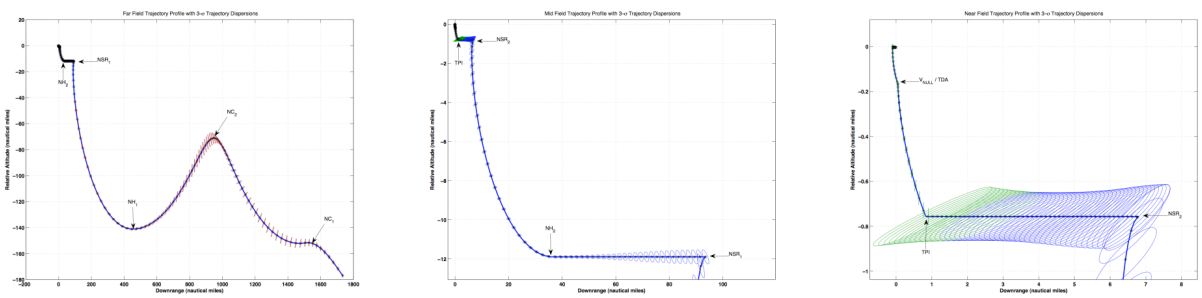

Figure 9: Position Dispersions as a Function of the Profile

In Figure 8, the maneuvers TDA and GLS are the Transfer to the Docking Axis and the Glideslope maneuvers, respectively. One can see that the dispersions, while large in the downrange direction, are well contained in the altitude direction and, on the final coelliptic segment have no possibility of intersecting with the target orbit, until the TPI maneuver is executed. In Figure 9, the trajectory dispersions change color to indicate the type of sensor in view - red ellipses indicate no direct relative sensing, blue indicates star tracker and RF based range and range-rate, and green indicates LIDAR sensing.

One can see, from these plots as well as from the more extensive analysis summarized here, that the Orion rendezvous phase of the mission meets its objectives of good relative navigation performance, good dispersion control and passive safety. In addition, the maneuver statistics are listed in Table 2, which shows good (i.e. small) $\Delta V$ statistics. 


\begin{tabular}{|c|c|c|c|}
\hline Maneuver Type & Maneuver Time & $\begin{array}{c}\text { Nominal } \Delta V \\
(\mathrm{ft} / \mathrm{s})\end{array}$ & $\begin{array}{l}\sigma_{\mathrm{GPS}} \\
(\mathrm{ft} / \mathrm{s})\end{array}$ \\
\hline$N C_{l}$ & $16^{\mathrm{m}}$ & 342.78 & 5.38 \\
\hline $\mathrm{NC}_{2} / \mathrm{NPC}$ & $1^{\mathrm{h}} 4^{\mathrm{m}}$ & 20.34 & 13.23 \\
\hline $\mathrm{NH}_{\mathrm{l}}$ & $1^{\mathrm{h}} 47^{\mathrm{m}}$ & 103.21 & 2.32 \\
\hline$N S R_{1}$ & $2^{\mathrm{h}} 33^{\mathrm{m}}$ & 225.42 & 5.10 \\
\hline $\mathrm{NH}_{2}$ & $3^{\mathrm{h}} 20^{\mathrm{m}}$ & 18.99 & 0.21 \\
\hline $\mathrm{NSR}_{2}$ & $4^{\mathrm{h}} 7^{\mathrm{m}}$ & 18.96 & 0.17 \\
\hline$T P I$ & $5^{\mathrm{h}} 26^{\mathrm{m}}$ & 3.36 & 5.97 \\
\hline$M C$ & $5^{\mathrm{h}} 41^{\mathrm{m}}$ & 0.25 & 3.17 \\
\hline$V_{N U L L}$ & $5^{\mathrm{h}} 52^{\mathrm{m}}$ & 0.08 & 0.07 \\
\hline TOTAL & & 733.39 & 33.27 \\
\hline$R S S \sigma$ & & & 16.09 \\
\hline
\end{tabular}

Table 2: Flight Day 1 Rendezvous Maneuver Statistics

\section{B. Proximity Operations and Docking Results}

Several closed-loop GN\&C Monte Carlo studies were conducted to assess GN\&C system performance during proximity operations and docking. Specifically, the purpose was to determine the pose accuracy required from the Vision Navigation System (VNS) inside of 50 feet, the acceptability of the long-range accuracy requirements of the VNS, and the point of closest approach to the ISS that can be expected from the baseline proximity operations trajectory in the case of a loss of translational control authority after the TPI burn. The studies focus on the CEV-toISS baseline proximity operations trajectory for docking with PMA2.

The first Monte Carlo study was a sensitivity study which sought to map the accuracy of individual components of the VNS pose solution to relative navigation accuracy during final approach along the V-bar (inside of 50 feet). The sensitivity study was used to iterate on the VNS pose accuracy specification until the relative navigation accuracy required at docking was satisfied, as allocated from the LIDS docking mechanism contact envelope. A subsequent Monte Carlo simulation consisting of a larger number of runs was conducted to verify that the VNS pose accuracy specification taken from the sensitivity study did indeed satisfy the relative navigation requirement at docking. The results are presented in Figures 10 and 11.
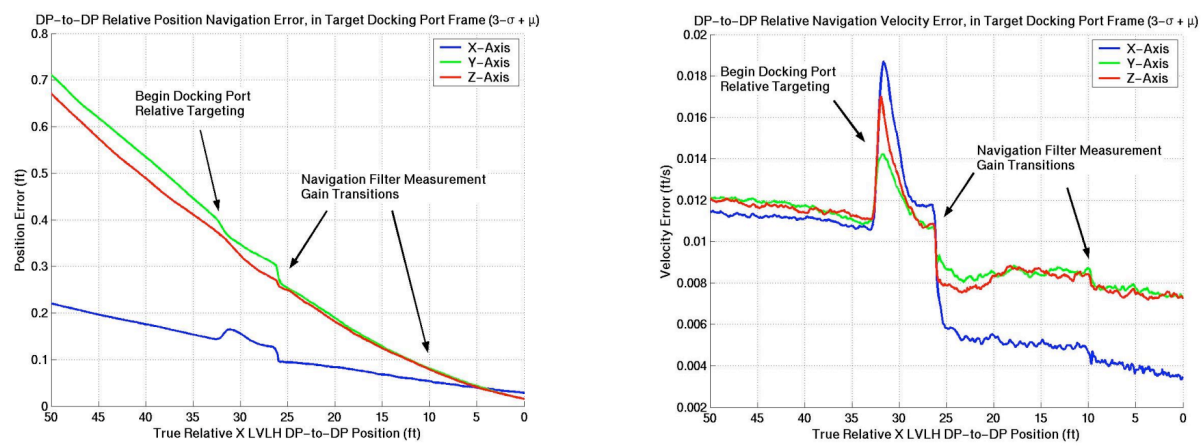

Figure 10: Proximity Operations Relative Navigation Position and Velocity Errors During Final 50 ft. of Approach Along V-bar

11

American Institute of Aeronautics and Astronautics 

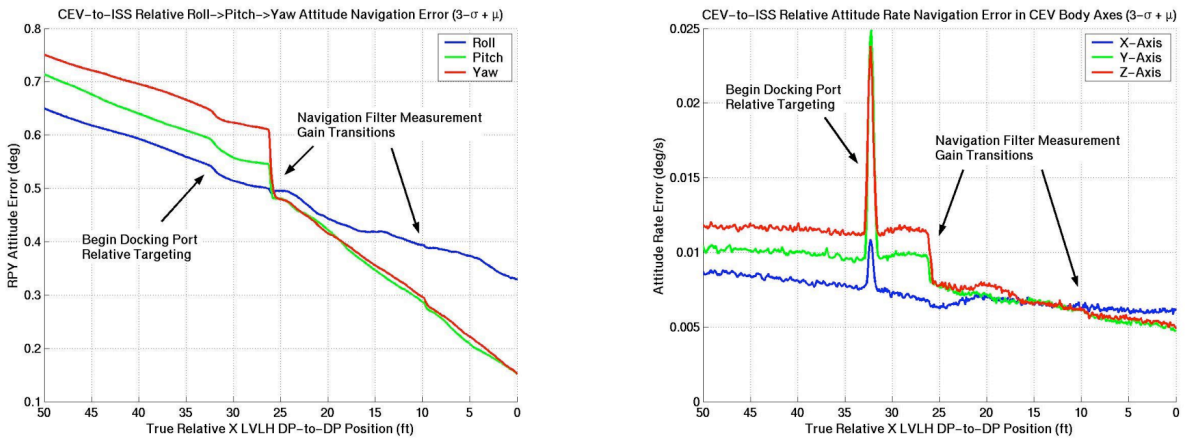

Figure 11: Proximity Operations Relative Navigation Attitude and Attitude Rate Errors During Final 50 ft. of Approach Along V-bar

The analysis showed that the adopted specification did meet the relative navigation accuracy requirements for docking. In fact, the VNS pose accuracy specification was designed so that the relative navigation accuracy requirements are met at 1 meter prior to contact, at which time the VNS sensor head is expected to be about 3 meters from its reflector array target on the ISS docking mechanism hatch. Note that misalignment of the VNS relative to the CEV docking mechanism frame was not modeled for this particular Monte Carlo simulation due to the fact that it had been accounted for separately in the relative navigation accuracy requirements at docking.

The second Monte Carlo study was intended to verify the acceptability of the long-range VNS accuracy specification. Though no RPOD requirement currently exists against which to judge the quantitative acceptability of the VNS range and bearing performance, a qualitative judgment can be made based on past experience and expected performance. With that in mind, a 1000-run Monte Carlo simulation was conducted with pre-TPI initial dispersions as specified by the aforementioned LINCOV rendezvous analysis, and the assumed VNS range and bearing accuracy specifications (this Monte Carlo study was conducted as a supplement to the LINCOV rendezvous study). The trajectory dispersions and fuel usage were then examined to check for obvious problems that might drive long range VNS specification values. All of the 1000 runs completed the proximity operations successfully, as can be seen from the trajectory hair plots in Figure 12.
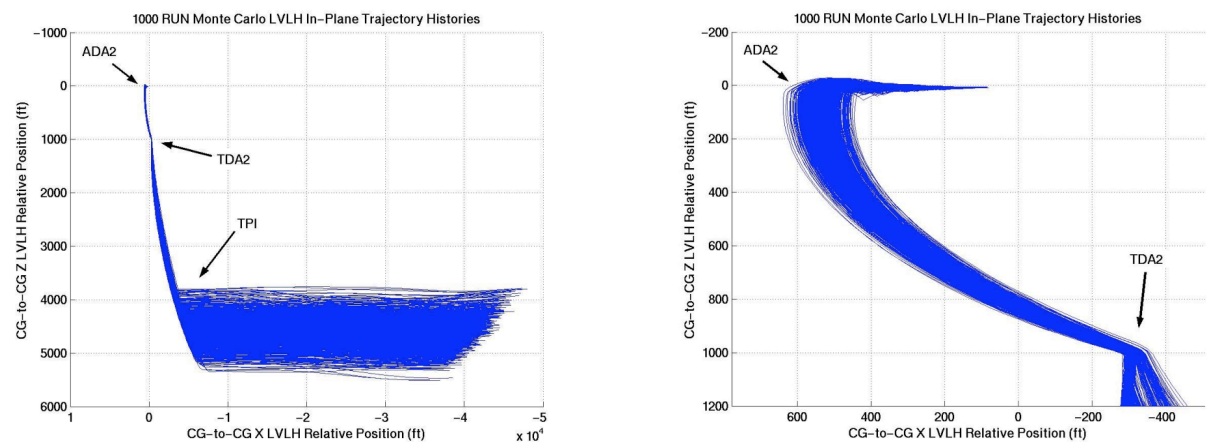

Figure 12: Proximity Operations Trajectory Dispersions; Overview and Close-Up on Post-TPI Segment 
A third Monte Carlo study was conducted to examine post-TPI dispersions under the assumption that the CEV lost all translational control authority after completing the TPI burn. The Monte Carlo analysis consisted of 1000 runs, each of which was propagated for a total time of 14,000 seconds to determine the trajectory behavior after the loss of translational control. Trajectory hair plots were used to assess the point of closest in-plane approach to the ISS, which turned out to be 875 feet. The in-plane trajectory dispersion and in-plane range hair plots are shown in Figure 13.
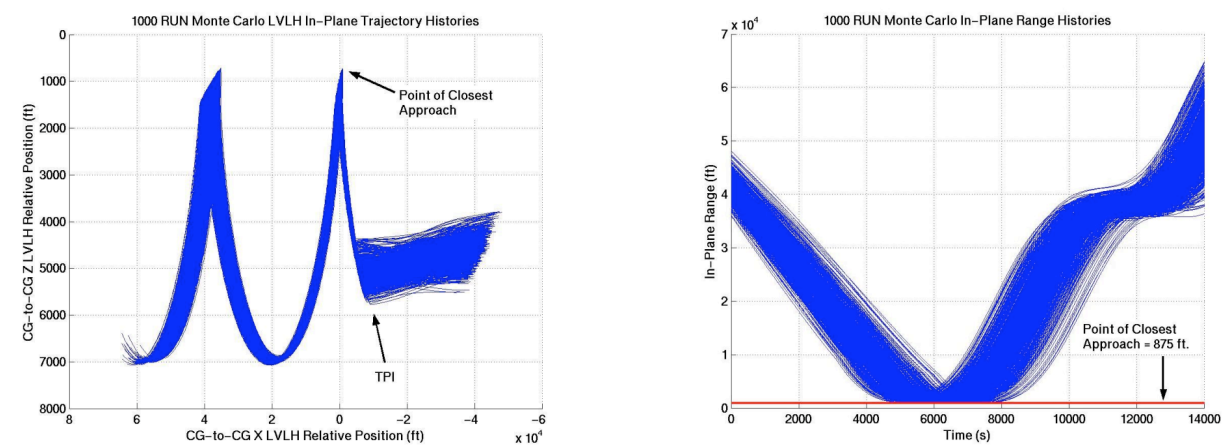

Figure 13: Post-NSR2 Histories Resulting From Post-TPI Loss of Translational Control Authority; In-Plane Trajectories and In-Plane Range Histories

\section{Conclusion}

This paper has described the Orion rendezvous, proximity operations and docking GNC design and analysis which has been performed to date. It has leveraged on RPOD experience over the past 40 years of manned space flight. In addition, it takes advantage of new sensor technology as well as meets the many constraints imposed on the RPOD GNC system. It is anticipated that the design will evolve as the vehicle takes shape.

\section{Acknowledgments}

The authors would like to thank Scott Cryan for providing the sensor concept of operations figure. The authors would also like to thank Jack Brazzel, Tim Crain, and David Saley for suggestions and many constructive comments that have improved the content and readability of the paper. In addition, the Lockheed-Martin RPOD team have been an integral part of the RPOD design maturation.

\section{References}

${ }^{1} \mathrm{Hu}, \mathrm{H}$. and Chambers, R., "Orion GN\&C Overview and Architecture," AIAA Guidance, Navigation, and Control Conference, AIAA-07-6678, Hilton Head, S.C., August 2007.

${ }^{2}$ Condon, J. and Buches, B., "Orion Mission Design and Analysis," AIAA Guidance, Navigation, and Control Conference, AIAA-07-6680, Hilton Head, S.C., August 2007.

${ }^{2}$ Brazzel, J, et. al., "NASA CEV On-orbit GN\&C Analysis," AAS Guidance and Control Conference, AAS-07-072, Breckenridge, CO, April 2007.

${ }^{3}$ Jackson, M., Gonzalez, R., Sims, C. "Orion Orbit Control Design and Analysis," AIAA Guidance, Navigation, and Control Conference, AIAA-2007-6684, Hilton Head, S.C., August 2007. 